\title{
Possibility of Detection of Exomoons with Inclined Orbits Orbiting Pulsar Planets Using the Time-of-Arrival Analysis
}

\author{
Antonio Pasqua ${ }^{1}$ and Khudhair A. Assaf ${ }^{2}$ \\ ${ }^{1}$ Department of Physics, University of Trieste, Via Valerio 2, 34127 Trieste, Italy \\ ${ }^{2}$ Department of Physics, Faculty of Science, University of Wasit, Wasit, Iraq \\ Correspondence should be addressed to Antonio Pasqua; toto.pasqua@gmail.com
}

Received 31 August 2013; Accepted 27 November 2013; Published 25 February 2014

Academic Editor: Gary Wegner

Copyright (c) 2014 A. Pasqua and K. A. Assaf. This is an open access article distributed under the Creative Commons Attribution License, which permits unrestricted use, distribution, and reproduction in any medium, provided the original work is properly cited.

\begin{abstract}
The perturbation caused by planet-moon binarity on the time-of-arrival (TOA) signal of a pulsar with an orbiting planet is derived for the case of the orbit of the planet-moon system inclined of an angle $\alpha$ with respect to the plane of the orbit of the planet-moon barycenter around the pulsar. We also consider both the orbits of the moon and the planet-moon barycenter as circular. The signal consists of three sinusoids with frequency, respectively, of $\left(2 n_{p}-3 n_{b}\right),\left(2 n_{p}-n_{b}\right)$, and $\left(2 n_{p}-3 n_{b}\right)$, where $n_{p}$ and $n_{b}$ are, respectively, the mean motions of the planet and moon around their barycenter and the planet-moon system around the host, respectively. The amplitude of the signal is equal to the fraction $\sin I\left[9(M p / M m) / 16(M p+M m)^{2}\right][r / R]^{5}\left(5 \sin ^{2} \alpha / 3-2 \sin \alpha / 3-2 \cos ^{2} \alpha / 9\right)$ of the system crossing time $R / c$, where $M_{p}$ and $M_{m}$ are, respectively, the mass of the planet and the mass of the moon, $r$ is their orbital separation, $R$ is the distance between the host pulsar and planet-moon barycenter, $I$ is the inclination of the orbital plane of the planet, and $c$ is the speed of light.
\end{abstract}

\section{Introduction}

Studies relative to the existence of planets external to our solar system have attracted a lot of attention in astronomy since long time.

In 1991, Bailes et al. of the Jodrell Bank Observatory of the University of Manchester announced the first ever pulsar planet detected orbiting the pulsar PSR 1829-10 [1]. However, this discovery was later retracted [2], shortly before the real detection of the first pulsar planets was announced. In fact, in 1992, Wolszczan and Frail announced the discovery of the first exoplanetary system (made by two different planets) orbiting the millisecond pulsar PSR 1257+12 [3]. These pulsar planets were the first two extrasolar planets discovered which have been confirmed as planets and also the first multiplanet extrasolar system observed (and of course the first pulsar planets discovered). Scientific community had several doubts concerning this discovery because of the retraction of the previous pulsar planet, and many questions arose about how pulsars could have planets. In fact, pulsars (or, equivalently, neutron stars) have been produced by the explosion of a supernova and it was widely thought that planets orbiting the star which underwent the supernova explosion would have been destroyed in the explosion itself. However, the existence of these pulsar planets was proved to be real. Then, it opened the problem (still under debate) of the explanation of their formation.

After these planets were observed by Wolszczan and Frail, in the following years scientists could observe other planets or minor bodies orbiting pulsars. Another additional planet of lower mass orbiting the same pulsar was later discovered.

In 2000, the millisecond pulsar PSR B1620-26 was found to have a circumbinary planet (i.e., PSR B1620-26 b) that orbits both it and its companion white dwarf, WD B1620-26 [4]. This was announced as the oldest planet ever discovered, since it is 12.6 billion years old. It is currently believed to have originally been the planet of WD B1620-26 before becoming a circumbinary planet, and therefore, while being discovered through the timing method, it did not form the way that PSR B1257+12's planets are believed to have.

In 2006, a circumstellar disk surrounding the magnetar (i.e., a pulsar with magnetic field higher than normal pulsars) 
$4 \mathrm{U} 0142+61$ was found, which is about 13,000 light years far from the Earth [5]. This disk is believed to have formed from the metal-rich debris left over from the supernova that leads to the formation of the pulsar and it is similar to those seen around Sun-like stars, which strongly suggests the possibility of formation of planets in a similar way.

In 2011, a planet which is thought to be the remaining core of a star that orbited a pulsar and orbiting the millisecond pulsar PSR J1719-1438 was announced [6]. This object represents a path to planetary status by evaporation of a star. It is estimated to have a density at least 23 times the density of water, a diameter of about $55,000 \mathrm{~km}$, a mass near that of Jupiter's, and an orbital period of 2 hours and 10 minutes when it is at a distance of $600,000 \mathrm{~km}$ from the pulsar. It is believed to be the diamond crystal core remaining from an evaporated white dwarf, with an estimated weight of $10^{31}$ carats.

PSR B1257+12D is an extrasolar object orbiting the pulsar PSR B1257+12, which is located in the Virgo constellation, 980 light years from the Earth. It is widely believed that this object is a big asteroid or a comet, located at a mean distance from the pulsar of 2,6 AU, with an orbital period of about 3,5 years. The perturbations produced by this body were referred to as a planet of mass similar to that of Saturn, that is, about 100 Earth masses, orbiting at a mean distance from the pulsar of about $40 \mathrm{AU}$; however, this discovery was not accepted and later on retracted. It is believed, then, that the observed perturbations are produced by a body of mass of the same order of an asteroid or a comet. If the presence of this small body orbiting around the pulsar will be confirmed, it will be the first example of asteroid or comet found outside the solar system. Some scientists also believe that this object can be the first and biggest of a series of objects which form an asteroid belt around the parent pulsar.

Then, currently, five exoplanets orbiting around three different pulsars have been discovered, three around PSR $1257+12$, one around PSR B1620-26, and one around PSR 17191438. These five planets include one with mass equivalent to 0.02 Earth masses, representing the exoplanet with the lowest mass known.

It must be also emphasized here that pulsar planets unlikely can harbor life as we know it, because the high levels of ionizing radiation emitted by the pulsar can prevent the formation of life and the visible light produced by pulsars is relatively low. Different can be the situation for moons orbiting the pulsar planets since the moons can be protected from the radiation produced by the pulsar by the planets themselves.

Since the discovery of the first exoplanet, more than one thousand exoplanets have been discovered (the website http://exoplanet.eu/ gives a continuous update of the data related to the extrasolar planets observed) until now. Most of them have masses bigger than Jupiter, which is the most massive planet of the Solar System. With the data obtained thanks to the new generation telescopes, it will be possible to find not only Earth-like planets but also exomoons orbiting exoplanets as well. As a result, the detectability of exomoons is starting to be explored in terms of the effects they are able to produce on planetary microlensing [7] and transit lightcurves $[8,9]$. Upper limits have already been placed on the mass and the radius of moons orbiting the planets HD $189733 \mathrm{~b}$ [10], HD 209458 b [11], and OGLE-TR-113 b [12]. Some other relevant works related to exomoons can be found in [13-25].

The detection of the first planet around a pulsar was obtained thanks to the investigation of periodic variations in the time-of-arrival (TOA) of the radio pulses emitted by the pulsar using a specific timing model. In fact, bodies orbiting a pulsar will produce regular changes in its pulsation, which can easily be observed from Earth. Since pulsars usually rotate with nearly constant period, changes produced by other bodies in the period itself can easily be detected with the help of precise timing measurements. Since the discovery of the first planets orbiting a pulsar, many efforts have been done in order to find more pulsar planets studying the behavior of many millisecond pulsars, with the results of the total discovery of the 5 planets mentioned above. In particular, we have that the planetary system orbiting the pulsar PSR $1257+12$ represents the only pulsar planet system with more than one planet. Given the timing stability of millisecond pulsars and the precision of the TOA method, the absence of additional discoveries of multiple systems and the few numbers of pulsar planets cannot be just an artifact of the method sensitivity. Miller and Hamilton [26] proposed that the scarcity of planets orbiting millisecond pulsars can be explained using the recycling hypothesis; that is, the accretion of matter from a donor star spins up the pulsar and makes it emit extremely stable radio signals. This type of accretion mechanism produces an X-ray luminosity which is sufficient to vaporize possible planets orbiting the parent pulsar. For this reason, Miller and Hamilton [26] suggested that PSR 1257+12 must represent a rare example of a high primordial spin. Even more problematic for scientists is to develop a reasonable physical mechanism which is able to explain reasonably the formation and the survival of the pulsar planets, either via survival of the supernova explosion that created the pulsar, by accretion from the disk resulting from the disruption of a companion, or by capturing from another main-sequence star [27]. We can therefore conclude that the rarity of pulsar planets indicates that the production of pulsar planets themselves is an exception rather than the general rule.

An example of timing model for the case in which the planet's orbit around the pulsar is circular is given by

$$
\begin{aligned}
\left(t_{N}-t_{0}\right)= & \left(T_{N}-T_{0}\right)+\Delta T_{\text {corr }} \\
& + \text { TOA }_{\text {pert }, p}\left(M_{s}, M_{p}, R, I, \phi_{b}(0)\right),
\end{aligned}
$$

where $t_{0}$ and $t_{N}$ are, respectively, the times at which the initial and $N$ th pulses are emitted in the pulsar's reference frame, $T_{0}$ and $T_{N}$ are, respectively, the times the initial and $N$ th pulses are received in the observer's reference frame, and the term $T_{\text {corr }}$ acts to change the frame of reference from the observer on Earth to the barycenter of the pulsar system (see [28] for more details on the components which form $\Delta T_{\text {corr }}$ ). The final term in (1) represents the effect produced by a planet on the motion of the pulsar. We have that, in this final term, $R$ indicates the distance between the pulsar and the planet, 
TABLE 1: Pulsar planets characteristics.

\begin{tabular}{lcccr}
\hline Pulsar & Planet & $M_{J}$ & $a$ & $P$ \\
\hline PSR B1620-26 & $\mathrm{b}$ & 2.5 & $23.0 \mathrm{AU}$ & 36525.0 days \\
PSR 1719-14 & $\mathrm{b}$ & 1.0 & $0.044 \mathrm{AU}$ & 0.09 day \\
PSR B1257+12 & $\mathrm{b}$ & $7 \times 10^{-5}$ & $0.19 \mathrm{AU}$ & 2003 \\
PSR B1257+12 & $\mathrm{c}$ & 0.013 & $0.36 \mathrm{AU}$ & 66.5419 days \\
PSR B1257+12 & $\mathrm{d}$ & 0.012 & $0.46 \mathrm{AU}$ & 98.2114 days \\
\hline
\end{tabular}

$I$ is the angle between the normal of the planet-pulsar orbit and the line-of-sight, and $\phi_{b}(0)$ is the initial angular position of the planet measured from the $x$-axis. Moreover, $M_{s}$ and $M_{p}$ are the mass of the pulsar and the mass of the planet, respectively.

The detection of low-mass planets around pulsars, in addition to measurements of orbital perturbations like the $2: 3$ orbital resonance between the planets PSR $1257+12 \mathrm{c}$ and PSR 1257+12d [29], shows the great sensitivity the TOA technique can reach, which makes it the best technique for moon detection now available. Furthermore, millisecond pulsars make optimal targets for high precision TOA observations due to their high rotation rate (which implies a large number of sampled pulses) and the low level of the noise activity [30].

In Table 1, it is possible to find the main information (i.e., the mass in Jupiter masses $M_{J}$, the semi major axis $a$ in astronomical units (AU), the period $P$ in days, and the year of discovery) of the five pulsar planets known up to now. More details about pulsar planets can be also found in [31-43].

The paper is organized in the following way. In Section 2, we derive the expression of the TOA produced by a hypothetical exomoon which orbits a pulsar planet in an inclined orbit. In Section 3, we make some considerations in order to understand under which conditions it is possible to detect signals produced by exomoons. Finally, in Section 4, we write the conclusions of this work.

\section{Calculation of the TOA Perturbation Caused by a Moon in Inclined Orbit}

Following the same procedure made by Lewis et al. [44], we now want to consider the effect produced by an orbit of the planet-moon system inclined by an angle $\alpha$ with respect to the plane containing the orbit of the planet-moon system around the pulsar. We still consider both orbits circular. For this purpose, the timing model given in (1) must be updated in order to include effects due to the presence of the moon and to the inclination of the moon orbit. An example model taking these assumptions into account is given by

$$
\begin{aligned}
\left(t_{N}-\right. & \left.t_{0}\right) \\
= & \left(T_{N}-T_{0}\right)+\Delta T_{\text {corr }} \\
& + \text { TOA }_{\text {pert }, p}\left(M_{s}, M_{p}+M_{m}, R, I, \phi_{b}(0)\right) \\
& + \text { TOA }_{\text {pert }, \mathrm{pm}}\left(M_{s}, M_{p}, M_{m}, R, r, I, \alpha, \phi_{b}(0), \phi_{p}(0)\right) .
\end{aligned}
$$

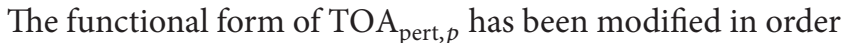
to indicate explicitly that it depends on the combined planetmoon mass and the term TOA $A_{\text {pert,pm }}$ is included in order to take into account the planet-moon binarity. $M_{m}$ represents the mass of the moon, $r$ is the separation between the planet and the moon, and $\phi_{p}(0)$ is the initial angular position of the planet measured from the planet-moon barycenter. $\alpha$ represents the inclination angle of the moon orbit, which is the angle between the normal of the planet-exomoon orbit and the line of sight. Moreover, the quantity $R$ represents, in this case, the distance between the pulsar and the planet-moon pair. The other quantities are defined as in (1). The expression of $\mathrm{TOA}_{\text {pert,pm }}$ can be derived from $\mathbf{R}_{s}$, which is the vector between the barycenter of the system and the pulsar, using the following relation:

$$
\frac{1}{c} \int_{0}^{t} \int_{0}^{t^{\prime}} \ddot{\mathbf{R}}_{s} \cdot \mathbf{n} d t^{\prime} d t=\mathrm{TOA}_{\mathrm{pert}, p}+\mathrm{TOA}_{\mathrm{pert}, \mathrm{pm}}
$$

where $c$ indicates the speed of light and $\mathbf{n}$ is a unitary vector pointing along the direction of the line of sight, which is the only direction along which quantities can be measured.

Equation (3) can be rewritten as the sum of the zeroth order term that describes the contribution to the signal given by $\mathrm{TOA}_{\text {pert, } p}$ and the tidal terms, describing the contributions to the signal given by $\mathrm{TOA}_{\text {pert,pm }}$ :

$$
\begin{aligned}
\frac{d^{2} \mathbf{R}_{s}}{d t^{2}}= & \frac{G\left(M_{p}+M_{m}\right)}{R^{3}} \mathbf{R} \\
+ & {\left[-\frac{G\left(M_{p}+M_{m}\right)}{R^{3}} \mathbf{R}\right.} \\
& \left.+\frac{G M_{p}\left(\mathbf{R}+\mathbf{r}_{p}\right)}{\left|\mathbf{R}+\mathbf{r}_{p}\right|^{3}}+\frac{G M_{m}\left(\mathbf{R}+\mathbf{r}_{m}\right)}{\left|\mathbf{R}+\mathbf{r}_{m}\right|^{3}}\right],
\end{aligned}
$$

where $\mathbf{R}_{s}, \mathbf{r}_{p}$, and $\mathbf{r}_{m}$ are defined as follows:

$$
\begin{gathered}
\mathbf{R}_{s}=-\frac{M_{m}+M_{p}}{M_{p}+M_{m}+M_{s}} \mathbf{R}, \\
\mathbf{r}_{p}=-\frac{M_{m}}{M_{p}+M_{m}} \mathbf{r}, \\
\mathbf{r}_{m}=\frac{M_{p}}{M_{p}+M_{m}} \mathbf{r} .
\end{gathered}
$$




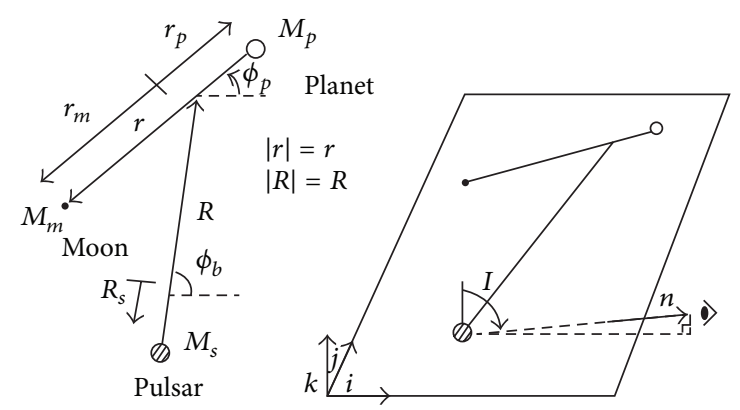

Figure 1: Distribution of the vectors used in this paper and the plan of the orbit containing the pulsar-planet plane.

The vectors $\mathbf{R}_{s}, \mathbf{R}, \mathbf{r}_{p}, \mathbf{r}_{m}$, and $\mathbf{r}$ are shown in Figure 1 . The terms inside the square brackets represent the contribution from the tidal terms.

Using the coordinate system represented in Figure 1, we can write

$$
\begin{aligned}
\mathbf{R}+\mathbf{r}_{m}= & {\left[R \cos \phi_{b}-\frac{M_{p}}{M_{p}+M_{m}} r \sin \alpha \cos \phi_{p}\right] \mathbf{i} } \\
& +\left[R \sin \phi_{b}-\frac{M_{p}}{M_{p}+M_{m}} r \sin \alpha \sin \phi_{p}\right] \mathbf{j} \\
& -\left[\frac{M_{p}}{M_{p}+M_{m}} r \cos \alpha \sin \phi_{p}\right] \mathbf{k}, \\
\mathbf{R}+\mathbf{r}_{p}= & {\left[R \cos \phi_{b}+\frac{M_{m}}{M_{p}+M_{m}} r \sin \alpha \cos \phi_{p}\right] \mathbf{i} } \\
& +\left[R \sin \phi_{b}+\frac{M_{m}}{M_{p}+M_{m}} r \sin \alpha \sin \phi_{p}\right] \mathbf{j} \\
& +\left[\frac{M_{m}}{M_{p}+M_{m}} r \cos \alpha \sin \phi_{p}\right] \mathbf{k},
\end{aligned}
$$

where $\mathbf{i}, \mathbf{j}$, and $\mathbf{k}$ are the unitary versors defining the $x, y$, and $z$ directions. In particular, $\mathbf{i}$ represents the direction along the line-of-sight, projected onto the plane of the orbit of the pulsar.

We have that, for circular orbits, $\phi_{p}(t)=n_{p} t+\phi_{p}(0)$ and $\phi_{b}(t)=n_{b} t+\phi_{b}(0)$, where $n_{b}$ and $n_{p}$ are the constant mean motions of the two respective orbits. Substituting (6) into the coefficients of the last two terms of (4), assuming that the condition $r \ll R$ is valid (which is usually respected), and using the binomial expansion to the order of $r^{2} / R^{2}$, we obtain the following quantities:

$$
\frac{G M_{m}}{\left|\mathbf{R}+\mathbf{r}_{m}\right|^{3}}=\frac{G M_{m}}{R^{3}}\left[1+\frac{3 M_{p}}{M_{m}+M_{p}} \frac{r}{R} \sin \alpha \cos \left(\phi_{b}-\phi_{p}\right)\right.
$$

$$
\begin{aligned}
& +\frac{M_{p}^{2}}{\left(M_{m}+M_{p}\right)^{2}} \frac{r^{2}}{R^{2}} \\
& \times\left(-\frac{3}{2} \sin ^{2} \alpha+\frac{15}{2} \sin ^{2} \alpha \cos ^{2}\left(\phi_{b}-\phi_{p}\right)\right. \\
& \left.\left.-\cos ^{2} \alpha \sin ^{2} \phi_{p}\right)\right] \\
& \frac{G M_{p}}{\left|\mathbf{R}+\mathbf{r}_{p}\right|^{3}}=\frac{G M_{p}}{R^{3}}\left[1-\frac{3 M_{m}}{M_{m}+M_{p}} \frac{r}{R} \sin \alpha \cos \left(\phi_{b}-\phi_{p}\right)\right. \\
& +\frac{M_{m}^{2}}{\left(M_{m}+M_{p}\right)^{2}} \frac{r^{2}}{R^{2}} \\
& \times\left(-\frac{3}{2} \sin ^{2} \alpha+\frac{15}{2} \sin ^{2} \alpha \cos ^{2}\left(\phi_{b}-\phi_{p}\right)\right. \\
& \left.\left.-\cos ^{2} \alpha \sin ^{2} \phi_{p}\right)\right]
\end{aligned}
$$

Substituting (6) and (7) into (4) yields, after some algebraic simplifications

$$
\begin{aligned}
\frac{d^{2} \mathbf{R}_{s}}{d t^{2}}= & \frac{G\left(M_{p}+M_{m}\right)}{R^{3}} \mathbf{R}+\frac{G M_{p} M_{m}}{\left(M_{p}+M_{m}\right)} \frac{r^{2}}{R^{4}} \\
\times & \left(-\frac{3}{2} \sin ^{2} \alpha+\frac{15}{2} \sin ^{2} \alpha\right. \\
& \left.\times \cos ^{2}\left(\phi_{b}-\phi_{p}\right)-\cos ^{2} \alpha \sin ^{2} \phi_{p}\right) \\
& \times\left(\cos \phi_{b} \mathbf{i}+\sin \phi_{b} \mathbf{j}\right. \\
& \left.\quad-\frac{M_{p}-M_{m}}{M_{p}+M_{m}} \frac{r}{R} \cos \alpha \sin \phi_{p} \mathbf{k}\right) \\
& -3 \sin \alpha \cos \left(\phi_{b}-\phi_{p}\right) \\
& \left.\times\left(\cos \phi_{p} \mathbf{i}+\sin \phi_{p} \mathbf{j}+\cos \alpha \sin \phi_{p} \mathbf{k}\right)\right]
\end{aligned}
$$

The vector $\mathbf{n}$ can be expressed as

$$
\mathbf{n}=\sin I \mathbf{i}+\cos I \mathbf{k} .
$$

Substituting (8) and (9) into (3) yields

$$
\begin{aligned}
\mathrm{TOA}_{\text {pert,pm }}= & -\sin I \frac{M_{p} M_{m}}{c\left(G M_{p}+M_{m}\right)} \frac{r^{2}}{R^{4}} \\
& \times\left[\frac{3}{4 n_{b}^{2}} \cos \phi_{b}\left(\sin ^{2} \alpha-2 \sin \alpha+2\right)\right.
\end{aligned}
$$




$$
\begin{aligned}
& +\frac{\left(15 \sin ^{2} \alpha-12 \sin \alpha-2 \cos ^{2} \alpha\right)}{8} \\
& \times \frac{\cos \left(\phi_{b}-2 \phi_{p}\right)}{\left(n_{b}-2 n_{p}\right)^{2}} \\
& +\frac{15}{8\left(3 n_{b}-2 n_{p}\right)^{2}} \sin ^{2} \alpha \cos \left(3 \phi_{b}-2 \phi_{p}\right) \\
& \left.-\cos ^{2} \alpha \frac{\cos \left(\phi_{b}+2 \phi_{p}\right)}{4\left(n_{b}+2 n_{p}\right)^{2}}\right] .
\end{aligned}
$$

Since we are considering the line-of-sight directed along $\mathbf{i}$, (10) gives only the contribution produced along this axis. The contribution produced along $\mathbf{k}$ is not considered here, also because it can be shown it is made by terms which are not detectable or negligible with respect to the contribution along i.

The $\cos \phi_{b}$ term in (10) has the same frequency as the signal of a lone planet and it is possible to demonstrate that it acts to increase the measured value of $M_{p}+M_{m}$ derived

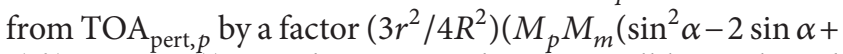
$\left.2) /\left(M_{p}+M_{m}\right)\right)$. For this reason, this term will be neglected as it will not be possible to detect it as a separate signal. Moreover, the edge of the stability region for a prograde satellite of the low-mass component of a high-mass binary can be approximated with $0.36 r_{H}$ for the case of circular orbits, where $r_{H}=R\left[M_{p} / 3 M_{s}\right]^{1 / 3}$ is the secondary's Hill radius [45], when the separation $r$ between planet and moon is equal to this maximum stable radius $n_{p} \approx 8 n_{b}$. As the limiting case $n_{b} \ll n_{p}$ is quite likely, the denominators of the terms we have derived will never approach zero. This fact, in addition to the assumption of circular orbit (i.e., eccentricity $e$ equal to zero), means that resonance effects can be safely neglected. Consequently, (10) can be simplified by neglecting $n_{b}$ in the denominators, which yields

$$
\begin{aligned}
\text { TOA }_{\text {pert,pm }}=- & \sin I \frac{G M_{p} M_{m}}{c\left(M_{p}+M_{m}\right)} \frac{r^{2}}{R^{4}} \\
\times & \frac{\left(15 \sin ^{2} \alpha-12 \sin \alpha-2 \cos ^{2} \alpha\right)}{32 n_{p}^{2}} \\
& \times \cos \left(\phi_{b}-2 \phi_{p}\right)+\frac{15}{32 n_{p}^{2}} \sin ^{2} \alpha \\
& \times \cos \left(3 \phi_{b}-2 \phi_{p}\right) \\
& \left.-\frac{\cos ^{2} \alpha}{16 n_{p}^{2}} \cos \left(\phi_{b}+2 \phi_{p}\right)\right] .
\end{aligned}
$$

Writing $n_{p}$ in terms of $r$ using the Kepler's law, (11) assumes the following form:

$$
\mathrm{TOA}_{\text {pert,pm }}=-\sin I \frac{M_{p} M_{m}}{\left(M_{p}+M_{m}\right)^{2}} \frac{R}{c}\left(\frac{r}{R}\right)^{5}
$$

$$
\begin{aligned}
& \times\left[\frac{\left(15 \sin ^{2} \alpha-12 \sin \alpha-2 \cos ^{2} \alpha\right)}{32}\right. \\
& \quad \times \cos \left(\phi_{b}-2 \phi_{p}\right)+\frac{15}{32} \sin ^{2} \alpha \cos \left(3 \phi_{b}-2 \phi_{p}\right) \\
& \left.\quad-\frac{\cos ^{2} \alpha}{16} \cos \left(\phi_{b}+2 \phi_{p}\right)\right],
\end{aligned}
$$

which is the final expression of the perturbation produced by the presence of an exomoon orbiting a pulsar planet.

\section{Possibility of Detection of Moons Orbiting Planet Pulsars}

In order to investigate which are the most favourable conditions to detect hypothetical moons orbiting pulsar planets, we simplify (12) by summing the amplitudes of the sinusoids, obtaining the following expression for $\mathrm{TOA}_{\mathrm{pert}, \mathrm{pm}}$ :

$$
\begin{aligned}
\max \left(\mathrm{TOA}_{\text {pert,pm }}\right)= & \frac{9 \sin I}{16} \frac{M_{m} M_{p}}{\left(M_{m}+M_{p}\right)^{2}} \frac{R}{c}\left(\frac{r}{R}\right)^{5} \\
& \times\left(\frac{5 \sin ^{2} \alpha}{3}-\frac{2 \sin \alpha}{3}-\frac{2 \cos ^{2} \alpha}{9}\right) .
\end{aligned}
$$

Then, for a fixed value of the ratio $r / R$, the value of the maximum amplitude of $\mathrm{TOA}_{\text {pert pm }}$ increases linearly with the distance $R$ between the parent pulsar and the planet-moon pair, which implies that the detection is more probable for planet-moon pairs which are as far as possible from the parent pulsars.

We can easily see that, in the limiting case corresponding to $\alpha=90^{\circ}$ (i.e., for coplanar orbits), we obtain that

$$
\left(\frac{5 \sin ^{2} \alpha}{3}-\frac{2 \sin \alpha}{3}-\frac{2 \cos ^{2} \alpha}{9}\right)=1
$$

Then, for $\alpha=90^{\circ}$, (13) reduces to

$$
\max \left(\mathrm{TOA}_{\text {pert }, p m}\right)=\frac{9 \sin I}{16} \frac{M_{m} M_{p}}{\left(M_{m}+M_{p}\right)^{2}} \frac{R}{c}\left(\frac{r}{R}\right)^{5},
$$

which is the same result obtained by Lewis et al. [44]. Lewis et al. [44] also found in their work that a stable exomoon orbiting the planet of the pulsar PSR B1610-26 can be hypothetically detected if the exomoon has a separation from the planet at least one-fiftieth of the separation of the planetmoon pair from the parent pulsar and a mass ratio to the planet of about $5 \%$ or larger.

We must also underline here that the results obtained by Lewis et al. [44] (which are recovered, as told, for the limiting case of $\alpha=90^{\circ}$ in our paper) are consistent with a similar work recently done by Schneider and Cabrera [46], who calculated the radial velocity perturbations produced on one component of a binary star system in the particular case the other component consists of an unresolved pair. Similar 
TABLE 2: TOA produced by hypothetical exomoons orbiting the known pulsar planets.

\begin{tabular}{|c|c|c|c|c|c|c|}
\hline Pulsar & Planet & $M_{J}$ & $a$ & $i$ & $\mathrm{TOA}_{1}$ & $\mathrm{TOA}_{2}$ \\
\hline PSR B1620-26 & $\mathrm{b}$ & 2.5 & $23 \mathrm{AU}$ & $50^{\circ}$ & $7412.42 \mathrm{~ns}$ & $21987.5 \mathrm{~ns}$ \\
\hline PSR 1719-14 & $\mathrm{b}$ & 1.0 & $0.0044 \mathrm{AU}$ & $50^{\circ}$ & $1.42 \mathrm{~ns}$ & $4.21 \mathrm{~ns}$ \\
\hline PSR B1257+12 & $\mathrm{b}$ & $7 \times 10^{-5}$ & $0.19 \mathrm{AU}$ & $50^{\circ}$ & $61.23 \mathrm{~ns}$ & $181.64 \mathrm{~ns}$ \\
\hline PSR B1257+12 & c & 0.013 & $0.36 \mathrm{AU}$ & $53^{\circ}$ & $120.96 \mathrm{~ns}$ & $337.94 \mathrm{~ns}$ \\
\hline PSR B1257+12 & $\mathrm{d}$ & 0.012 & $0.46 \mathrm{AU}$ & $47^{\circ}$ & $141.54 \mathrm{~ns}$ & $419.84 \mathrm{~ns}$ \\
\hline
\end{tabular}

results between Lewis et al. and Schneider and Cabrera are obtained if the radial velocity perturbation obtained in the work of Schneider and Cabrera is converted to a timing perturbation and the masses of the planet and moon are considered equal, as in the case investigated by Schneider and Cabrera [46] (it must be also noticed that $r$ in the work of Lewis et al. is equivalent to $2 a_{A}$ in the work of Schneider and Cabrera). We can also conclude that the results we obtained in this work are in agreement with the results of Schneider and Cabrera in the limiting case of $\alpha=90^{\circ}$.

We must also consider that, in the particular case of the pulsar PSR B1620-26 (which forms a binary system with a white dwarf), the perturbation signal does not match exactly the signals obtained using (13) and (15) due to the effects produced by the white dwarf companion, which introduces additional perturbations on both $\mathrm{TOA}_{\text {pert, } p}$ and $\mathrm{TOA}_{\text {pert,pm }}$. For this reason, the signal produced with our model represents an order of magnitude of the minimum detectable signal produced by an exomoon.

We now want to make some considerations on signals produced by hypothetical pulsar-moon pairs orbiting parent pulsars.

In the work of Lewis et al. [44], it was obtained, using the TOA model obtained by the authors, that a binary system made by two planets with separation of $0.1 \mathrm{AU}$ and with the same mass (chosen as equivalent to the mass of Jupiter $M_{J}$ ) located at a distance of 5.2 AU from the parent pulsar can produce a TOA ${ }_{\text {pert,pm }}$ with an amplitude of $960 \mathrm{~ns}$.

As an example, we now want to calculate the signals produced by exomoons orbiting the pulsar planets listed in Table 1, considering the expression of $\mathrm{TOA}_{\text {pert,pm }}$ we found in (13). As values of the distance $R$, we have considered the values of the semimajor axis $a$ of the planetary orbits (even if it is an approximation since $R$ is referred to as a circular orbit while a as an elliptical orbit). As inclination angle $\alpha$ of the moon orbit, we have chosen the value of $\alpha=80^{\circ}$. Moreover, we considered two different cases for the distance $r$ between the planet and the moon and moon mass $M_{m}$. In the first one, as separation between the planet and the moon, we have chosen $r=R / 35$ and a moon mass $M_{m}$ which is one-tenth of the planet mass. In the second case, we have considered $r=R / 30$ and a moon mass which is 0.15 times the mass of the planet. We must also emphasize here some aspects considered during the calculations done in order to obtain TOA $_{\text {pert,pm }}$ for the pulsar planets. The angles $i$, as well as the other pulsar planets information, are obtained from the website http://exoplanet.eu/catalog/. As it is possible to see in the page with pulsar planet data, not all the inclinations for pulsar planets are available (to be more precise, only those of PSR B1257+12c and PSR B1257+12d have been obtained thanks to observations). For all the other planets, we considered a value of $i=50^{\circ}$. We must underline here that, in the website with exoplanets data as well as in other works related to this field, the letter $i$ is often used in order to indicate the angle between the normal of the planet-pulsar orbit and the line-of-sight while in our work we used $I$. The values of the $\mathrm{TOA}_{\text {pert,pm }}$ in nanoseconds for the five known pulsar planets of the two examples considered are given in the last two columns of Table 2 (in particular, the first column corresponds to the case with $r=R / 35$ while the second column corresponds to the case with $r=R / 30$ ). As we can clearly see from the results obtained, we have that $\mathrm{TOA}_{\text {pert,pm }}$ increases with the increasing of the value of $R$, confirming the result discussed above; that is, for a given value of the ratio $r / R$, the expression of TOA $_{\text {pert,pm }}$ increases linearly with the value of $R$.

We also plotted the expression of $\mathrm{TOA}_{\text {pert,pm }}$ derived in (13) for different values of the parameters involved. We have chosen $\sin I=1$, which represents the case the plane containing the planet orbit around the pulsar is edge-on (this assumption represents also the case which produces the strongest signal according to (13) and then the signal easier to detect), a mass of the planet in the range $(0.5 \div 3) M_{J}$, a mass of the moon which is one-tenth of the planet mass, and an inclination angle $\alpha$ of the moon orbit in the range $\left(50^{\circ} \div 90^{\circ}\right)$. We have considered two different cases for $R$ : in the first one, we have chosen $R=5.2 \mathrm{AU}$, while in the second one we have chosen $R=23 \mathrm{AU}$, which is the value of the semimajor axis of the planet PSR B1620-26b. In both cases, we have considered $r=R / 40$. In Figure 2, we have plotted the case corresponding to $R=5.2 \mathrm{AU}$. We can clearly see that the level of TOA $\mathrm{A}_{\text {pert,pm }}$ goes from about half microsecond to a value of about one microsecond or a bit higher than one microsecond. Instead, the case corresponding to $R=23 \mathrm{AU}$ has been plotted in Figure 3. We can derive from this figure that the level of $\mathrm{TOA}_{\text {pert,pm }}$ is of the order of few microseconds. In both Figures 2 and 3, the intensity of the signal increases with the increasing of the angle $\alpha$, reaching the maximum value for $\alpha=90^{\circ}$, which is the limiting case we studied above corresponding to the model of Lewis et al. [44].

In order to have a better idea of the strength of the exomoons signals we found in our examples, we must also remember that pulsar planets produce signals ranging from microseconds to milliseconds (depending on their distance from the parent pulsar and their mass), then usually bigger 


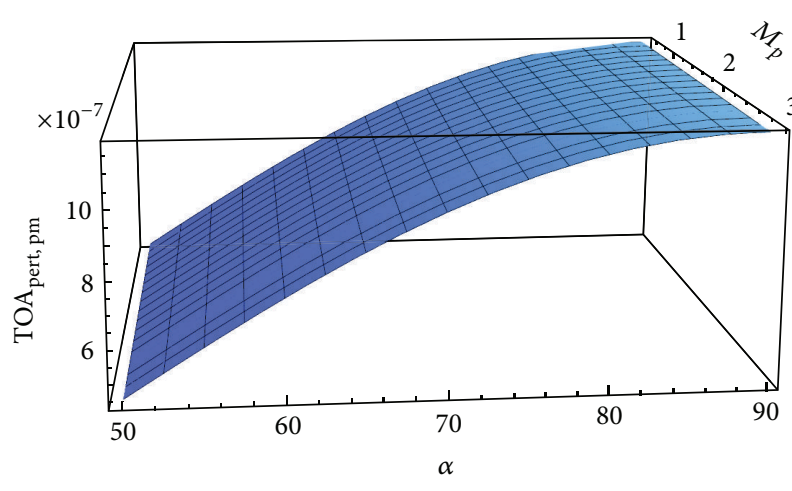

Figure 2: Values of TOA $A_{\text {pert,pm }}$ in seconds obtained from (13) for a range of values of the planet mass $M_{p}$ and moon orbit inclination $\alpha$. We have also chosen $\sin I=1, R=5.2 \mathrm{AU}, r=R / 40$, and $M_{m}=$ $M_{p} / 10$

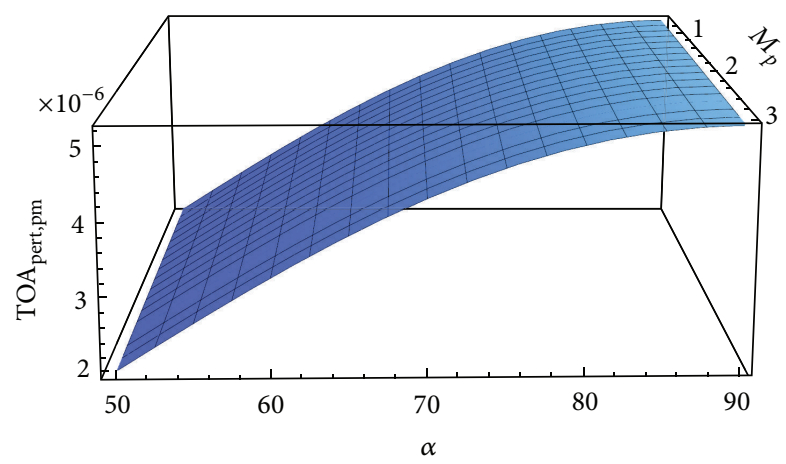

FIgURE 3: Values of TOA $A_{\text {pert,pm }}$ in seconds obtained from (13) for a range of values of the planet mass $M_{p}$ and moon orbit inclination $\alpha$. We have also chosen $\sin I=1, R=23 \mathrm{AU}, r=R / 40$, and $M_{m}=$ $M_{p} / 10$.

than those we found in Table 2 and Figures 2 and 3 relative to exomoons.

We can now make some speculation about the situation corresponding to $M_{m}=M_{p}$, which represents a system of two identical planetary objects with the same mass orbiting around a common center of gravity and also orbiting around the parent pulsar. Such a system was never observed up to now, but we also know exoplanets have many surprising features (like the metallicity of parent stars higher than that of our Sun and distances from the parents stars less than the distance between Mercury and the Sun); then we cannot exclude a priori that these systems can exist. We must underline that, in the limiting case of $M_{p}=M_{m}$, it is not proper to call one of the objects moon, but we are in the case of a binary system made by two planets of equivalent mass. We must also emphasize that it is not perfectly right to consider the orbits of these two planets around their center of mass as circular, but the proper case is to consider elliptical orbits. Anyway, the main aim of this paper is to give an order of magnitude of the effect produced by the system we are studying, and considering circular orbits, even if not completely correct, can give some useful information on the signal produced by hypothetical exomoons. Furthermore, many authors of works related to exoplanets and exomoons often consider circular orbits for binary systems with objects of the same mass, as it was also made in the example of Lewis et al. [44] (which is also indicated in this work) and also done by Schneider and Cabrera [46]. Other physical effects, like planet-planet interactions, produced by the strong gravitational field of the parent pulsar, tidal forces, and eccentricity of the orbits can be discussed in future works in order to understand how they affect the signals produced by exomoons.

We have that (13), in the limiting case of $M_{m}=M_{p}$, reduces to

$$
\begin{aligned}
\max \left(\text { TOA }_{\text {pert,pm }}\right)= & \frac{9 \sin I}{64} \frac{R}{c}\left(\frac{r}{R}\right)^{5} \\
& \times\left(\frac{5 \sin ^{2} \alpha}{3}-\frac{2 \sin \alpha}{3}-\frac{2 \cos ^{2} \alpha}{9}\right),
\end{aligned}
$$

where we used the fact that, for $M_{m}=M_{p}, M_{m} M_{p} /$ $\left(M_{m}+M_{p}\right)^{2}=1 / 4$.

Instead, (15), in the limiting case corresponding to $M_{m}=$ $M_{p}$, reduces to

$$
\max \left(\mathrm{TOA}_{\text {pert,pm }}\right)=\frac{9 \sin I}{64} \frac{R}{c}\left(\frac{r}{R}\right)^{5}
$$

We can consider another limiting case. In fact, for an angle $\alpha$ approximately of $47^{\circ} 24^{\prime} 28^{\prime \prime}$, the quantity $\left(5 \sin ^{2} \alpha / 3-\right.$ $\left.2 \sin \alpha / 3-2 \cos ^{2} \alpha / 9\right)$ is equal to zero, and then the expression of the maximum TOA $\mathrm{pert}_{\text {pm }}$ given in (13) becomes zero too, which means there is no perturbative contribution from the presence of the exomoon; then we are able to detect only the signal produced by the planet.

Unfortunately, there are some practical limits in the TOA technique for the detection of an exomoon. First of all, other systems which are able to produce similar signals need to be investigated in more details in order that they can be distinguished from signal produced by exomoons. Possible processes include unmodelled interactions between planets [47], gravitational waves emission [48], periodic variation in the interstellar medium [49], the hypothetical presence of other small planets or minor bodies, and pulsar precession [50]. Future improvements of our work can be done taking into account elliptic orbits for planet and/or moon orbits.

Second, the suitability of pulsars for signal detection is limited due to two main noise sources, that is, phase jitter and red timing noise (for more details, see [30]). Phase jitter is an error due to pulse-to-pulse variations which leads to statistically independent errors in TOA measurements. Phase jitter decreases with the increasing of the rotation rate (i.e., decreasing period $P$ of the pulsar), due to the resultant increase in the number of pulses sampled in each integration. Instead, red timing noise refers to noise for which neighbouring TOA residuals are correlated. Red timing noise, which strongly depends on the time derivative of period of the pulsar $\dot{P}$, has been historically modeled as a random 
walk in phase, frequency, or frequency derivative (see [51-54] for more details). In order to illustrate the effects produced by the two noise sources described above on the TOA method precision, an estimate of their combined residuals as a function of the pulsar period $P$ of the pulsar and of the time derivative of the pulsar period $\dot{P}$ was shown in Figure 3 of the paper of Lewis et al. [44], which is based on Figure 9 of the paper of Cordes [30]. From Figure 3 of the paper of Lewis et al. [44], it can be clearly obtained that the noise level increases with the increasing of the pulsar period $P$, going from some nanoseconds for millisecond pulsars to few millisecond, for pulsars with periods of the order of a second or a bit less than one second. Since the correlated timing noise measured for each individual pulsar can vary from the predicted values indicated in Figure 3 of Lewis et al. paper even of two orders of magnitude (see the work of Arzoumanian et al. [55] in order to have more information), the results obtained about the pulsar noise have as main aim to demonstrate the general pulsar noise properties; then it is not intended to precisely predict individual pulsar noise physical characteristics and behavior. The results shown in Figure 3 of Lewis et al. also justify the choice of millisecond pulsars as possible targets for detections of exomoons, since their noise is lower than the signal produced by the hypothetical presence of the exomoon, as we obtained thanks to the example we considered. Going to upper periods, the noise level becomes more relevant, which makes the exomoon detection more difficult and challenging (and in long period pulsars case practically impossible). We can also easily understand, then, that one of the main limits of the TOA technique is represented by the level of the noise produced by the mechanism we described: less is the noise level, better are the observations we can make, and therefore more efficient is the TOA technique.

Third, the possibility moons will be detected depends on whether or not they exist in certain particular configurations, which depend on their formation history and orbital stability. Recent researches suggest that there are physical mass limits for satellites of both gas giants [56] and terrestrial planets [57]. Also, tidal and three body effects can strongly affect the stability and lifetime of exomoons [58-60].

It must be also remembered that, while this method was investigated for the specific case of a pulsar planet, the TOA technique can also be applied to planets orbiting other clocklike hosts, like pulsating giant stars [61] and white dwarfs [62].

\section{Conclusions}

In this work, we calculated the effect produced by an exomoon orbiting a pulsar planet, considering the orbit of the exomoon inclined of an angle $\alpha$ with respect to the orbit of the planet around the pulsar itself. This work can be considered as an extension of the work of Lewis et al. [44] since they considered the orbit of the moon and orbit of the planet around the pulsar coplanar. We considered both orbits as circular. We found that the perturbation caused by the moon implies the presence of a multiplicative factor $\left(5 \sin ^{2} \alpha / 3-\right.$ $\left.2 \sin \alpha / 3-2 \cos ^{2} \alpha / 9\right)$ in the signal also obtained in the work of Lewis et al. In the limiting case of $\alpha=90^{\circ}$, that is, coplanar orbits, we obtain that the multiplicative factor is equal to one and the result of Lewis et al. is recovered.

We also considered some examples in order to understand which is the level of the signals produced by exomoons and if it is possible to detect them. Lewis et al. [44] derived that a stable exomoon orbiting the planet PSR B1610-26b can be detected in the case the exomoon has a distance from the planet which is at least one-fiftieth of the distance of the planet from the parent pulsar and the mass of the moon is about $5 \%$ of the planet mass or larger.

As also discussed in the work of Lewis et al. [44], we have that a binary system made by two planets with a separation of $0.1 \mathrm{AU}$ and with the same mass (equivalent to the Jupiter mass $M_{J}$ ) located at a distance of $5.2 \mathrm{AU}$ from the parent pulsar is able to produce a signal which has amplitude of $960 \mathrm{~ns}$.

Moreover, we also considered the case corresponding to a mass of the moon equal to the mass of the planet, which is a particular case not yet observed but which cannot be excluded a priori. Furthermore, we have found that, for an inclination angle $\alpha$ approximately of $47^{\circ} 24^{\prime} 28^{\prime \prime}$, there is no contribution produced by the presence of the moon since $\mathrm{TOA}_{\text {pert,pm }}=0$.

Applying to the five known pulsar planets the $\mathrm{TOA}_{\text {pert,pm }}$ model we derived in (13), we have obtained the level of the signal that a hypothetical exomoon orbiting the respective pulsar planet can produce. As expected, we have found that, for fixed values of $r / R$, the amplitude of $\mathrm{TOA}_{\text {pert,pm }}$ increases with $R$. In particular, we have found that, for planets PSRR $1719-14 b$, PSR B1257+12b, and PSR B1257+12c, the signal produced by the exomoons is difficult to be detected since it is of the same order as or lower than the noise level. For PSR B1257+12d, instead, we are able to detect a signal if $R$ is large enough. The strongest signal is produced by the exomoon orbiting PSR B1620-26, since it is the case with higher $R$, that is, 23 astronomical units.

We also plotted the expression of $\mathrm{TOA}_{\text {pert,pm }}$ derived in (13) considering the two cases corresponding to $R=5.2 \mathrm{AU}$ and $R=23 \mathrm{AU}$ (which is the distance of the planet PSR B1620-26 from the parent pulsar) along with an inclination angle $\alpha$ in the range $\left(50^{\circ} \div 90^{\circ}\right)$, a mass of the planet in the range $(0.5 \div 3) M_{J}$, and a mass of the moon one-tenth of the planet mass: we obtained a signal of the order of a microsecond (or a bit less) for $R=5.2 \mathrm{AU}$ and a signal of the order of few microseconds in the case of $R=23 \mathrm{AU}$.

As discussed, the signal we obtained is just an order of magnitude of possible signals produced by exomoons, since there are many physical factors which must be taken into account in order to be more precise.

Future improvements of this work can be done considering more precise details, for example, elliptical orbits of the planet around the parent pulsar and/or the moon orbiting the planet.

\section{Conflict of Interests}

The authors declare that there is no conflict of interests regarding the publication of this paper. 


\section{References}

[1] M. Bailes, A. G. Lyne, and S. L. Shemar, "A planet orbiting the neutron star PSR1829-10," Nature, vol. 352, no. 6333, pp. 311-313, 1991.

[2] A. G. Lyne and M. Bailes, "No planet orbiting PS R1829-10," Nature, vol. 355, no. 6357, p. 213, 1992.

[3] A. Wolszczan and D. A. Frail, "A planetary system around the millisecond pulsar PSR1257+ 12," Nature, vol. 355, no. 6356, pp. 145-147, 1992.

[4] D. C. Backer, R. S. Fostert, and S. Sallmen, "A second companion of the millisecond pulsar 1620-26," Nature, vol. 365, no. 6449, pp. 817-819, 1993.

[5] Z. Wang, D. Chakrabarty, and D. L. Kaplan, "A debris disk around an isolated young neutron star," Nature, vol. 440, no. 7085, pp. 772-775, 2006.

[6] M. Bailes, S. D. Bates, V. Bhalerao et al., "Transformation of a star into a planet in a millisecond pulsar binary," Science, vol. 333, no. 6050, pp. 1717-1720, 2011.

[7] C. Han and W. Han, "On the feasibility of detecting satellites of extrasolar planets via microlensing," The Astrophysical Journal, vol. 580, no. 1, p. 490, 2002.

[8] P. Sartoretti and J. Schneider, "On the detection of satellites of extrasolar planets with the method of transits," Astronomy and Astrophysics Supplement Series, vol. 134, no. 3, pp. 553-560, 1999.

[9] G. M. Szabó, K. Szatmáry, Z. Divéki, and A. Simon, "Possibility of a photometric detection of 'exomoons," Astronomy \& Astrophysics, vol. 450, no. 1, pp. 395-398, 2006.

[10] F. Pont, R. L. Gilliland, C. Moutou et al., "Hubble Space Telescope time-series photometry of the planetary transit of HD 189733: no moon, no rings, starspots," Astronomy \& Astrophysics, vol. 476, no. 3, pp. 1347-1355, 2007.

[11] T. M. Brown, D. Charbonneau, R. L. Gilliland, R. W. Noyes, and A. Burrows, "Hubble space telescope time-series photometry of the transiting planet of HD 209458," Astrophysical Journal Letters, vol. 552, no. 2, pp. 699-709, 2001.

[12] M. Gillon, F. Pont, C. Moutou et al., "High accuracy transit photometry of the planet OGLE-TR-113b with a new deconvolution-based method," Astronomy \& Astrophysics, vol. 459, no. 1, pp. 249-255, 2006.

[13] S. Awiphan and E. Kerins, "The detectability of habitable exomoons with Kepler," Monthly Notices of the Royal Astronomical Society, vol. 432, no. 3, pp. 2549-2561, 2013.

[14] T. Sasaki, J. W. Barnes, and D. P. O’Brien, "Outcomes and duration of tidal evolution in a star-planet-moon system," The Astrophysical Journal, vol. 754, no. 1, p. 51, 2012.

[15] A. E. Simon, G. M. Szabó, L. L. Kiss, and K. Szatmáry, "Signals of exomoons in averaged light curves of exoplanets," American Astronomical Society, vol. 419, no. 1, pp. 164-171, 2012.

[16] D. M. Kipping, "The search for exomoons," in Proceedings of the Molecules in the Atmospheres of Extrasolar Planets, vol. 450 of Astronomical Society of the Pacific Conference Series, p. 47, 2011.

[17] K. M. Lewis, The detectability of moons of extra-solar planets [Ph.D. thesis], 2011.

[18] D. M. Kipping, "An algorithm for generating dynamic planetmoon transits," Monthly Notices of the Royal Astronomical Society, vol. 416, no. 1, pp. 689-709, 2011.

[19] D. M. Kipping, S. J. Fossey, G. Campanella, J. Schneider, and G. Tinetti, "Pathways towards habitable planets," in Proceedings of the International Conference Organized by the Spanish National Research Council, C. D. F. Vincent, D. M. Gelino, and I. Ribas,
Eds., Astronomical Society of the Pacific Conference Series, p. 139, Astronomical Society of the Pacific, Barcelona, Spain, September 2009.

[20] C. Liebig and J. Wambsganss, "Detectability of extrasolar moons as gravitational microlenses," Astronomy \& Astrophysics, vol. 520, article A68, 2010.

[21] A. E. Simon, G. M. Szabó, K. Szatmáry, L. L. Kiss, and R. M. Not, "Methods for exomoon characterization: combining transit photometry and the Rossiter-McLaughlin effect," Astronomical Society, vol. 406, no. 3, pp. 2038-2046, 2010.

[22] J. R. Donnison, "The Hill stability of the possible moons of extrasolar planets," Monthly Notices of the Royal Astronomial Society, vol. 406, pp. 1918-1934, 2010.

[23] L. Kaltenegger, "Characterizing habitable exomoons," Astrophysical Journal Letters, vol. 712, no. 2, pp. L125-L130, 2010.

[24] D. M. Kipping, S. J. Fossey, and G. Campanella, "On the detectability of habitable exomoons with Kepler-class photometry," Monthly Notices of the Royal Astronomical Society, vol. 400, no. 1, pp. 398-405, 2009.

[25] A. E. Simon, G. M. Szabó, and K. Szatmáry, "Exomoon simulations," Earth Moon and Planets, vol. 105, no. 2-4, pp. 385-389, 2009.

[26] M. C. Miller and D. P. Hamilton, "Implications of the PSR $1257+12$ planetary system for Isolated millisecond pulsars," The Astrophysical Journal, vol. 550, no. 2, p. 863, 2001.

[27] S. Sigurdsson, "Genesis of a planet in Messier 4," Astrophysical Journal Letter, vol. 415, no. 1, pp. L43-L46, 1993.

[28] D. C. Backer, "A pulsar timing tutorial and NRAO Green Bank observations of PSR 1257+12," in Proceedings of the Conference Planets around Pulsars, pp. 11-18, California Institute of Technology, May 1992.

[29] M. Konacki and A. Wolszczan, "Masses and orbital inclinations of planets in the PSR B1257+12 System," Astrophysical Journal Letters, vol. 591, no. 2, pp. L147-L150, 2003.

[30] J. M. Cordes, "The detectability of planetary companions to radio pulsars," in Proceedings of the Conference Planets around Pulsars, pp. 43-60, California Institute of Technology, May 1992.

[31] A. Wolszczan, Pulsar Timing, General Relativity and the Internal Structure of Neutron Stars, vol. 101, 1999.

[32] A. Wolszczan, "Compact stars in binaries," in Proceedings of the Compact Stars in Binaries, J. van Paradijs, E. P. J. van den Heuvel, and E. Kuulkers, Eds., vol. 165 of IAU Symposium, p. 187, Kluwer Academic, August 1994, As Part of the 22nd General Assembly of the IAU in The Hague, the Netherlands.

[33] A. Wolszczan, "Applications of pulsar timing," Acta Cosmologica, vol. 23, pp. 127-130, 1997.

[34] A. Wolszczan, Planets Beyond the Solar System and the Next Generation of Space Missions, vol. 119, 1997.

[35] A. Wolszczan, "Discovery of two planets around a millisecond pulsar," in LPI Contributions, vol. 781, p. 53, the Lunar and Planetary Institute, Houston, Tex, USA, March 1992, Press Abstracts for the Twenty-third Lunar and Planetary Science Conference.

[36] A. Wolszczan, Lunar and Planetary Institute Science Conference Abstracts, vol. 23, 1992.

[37] A. Wolszczan, Planets Around Pulsars, vol. 36, 1993.

[38] A. Wolszczan, Millisecond Pulsars. A Decade of Surprise, vol. 72, 1995.

[39] A. Wolszczan, "Pulsar planets: status of observations and understanding," American Astronomical Society Meeting Abstracts, vol. 221, p. 424.01, 2013. 
[40] A. Wolszczan, "Discovery of pulsar planets," New Astronomy Reviews, vol. 56, no. 1, pp. 2-8, 2012.

[41] A. Wolszczan, "Confirmation of earth-mass planets orbiting the millisecond pulsar PSR B1257 + 12," Science, vol. 264, no. 5158, pp. 538-542, 1994.

[42] S. Sigurdsson, H. B. Richer, B. M. Hansen, I. H. Stairs, and S. E. Thorsett, "A young white dwarf companion to pulsar B1620-26: evidence for early planet formation," Science, vol. 301, no. 5630, pp. 193-196, 2003.

[43] M. Bailes, S. D. Bates, V. Bhalerao et al., "Transformation of a star into a planet in a millisecond pulsar binary," Science, vol. 333, no. 6050, pp. 1717-1720, 2011.

[44] K. M. Lewis, P. D. Sackett, and R. A. Mardling, "Possibility of detecting moons of pulsar planets through time-of-arrival analysis," The Astrophysical Journal Letters, vol. 685, no. 2, p. L153, 2008.

[45] M. J. Holman and P. A. Wiegert, "Long-term stability of planets in binary systems," Astronomical Journal, vol. 117, no. 1, pp. 621$628,1999$.

[46] J. Schneider and J. Cabrera, "Can stellar wobble in triple systems mimic a planet?” Astronomy \& Astrophysics, vol. 445, no. 3, pp. 1159-1163, 2006.

[47] G. Laughlin and J. E. Chambers, "Short-term dynamical interactions among extrasolar planets," The Astrophysical Journal Letters, vol. 551, no. 1, p. L109, 2001.

[48] S. Detweiler, "Pulsar timing measurements and the search for gravitational waves," Astrophysical Journal, vol. 234, pp. 11001104, 1979.

[49] K. Scherer, H. Fichtner, J. D. Anderson, and E. L. Lau, "A pulsar, the heliosphere, and pioneer 10: probable mimicking of a planet of PSR B1257 + 12 by solar rotation," Science, vol. 278, no. 5345, pp. 1919-1921, 1997.

[50] T. Akgün, B. Link, and I. Wasserman, "Precession of the isolated neutron star PSR B1828-11," Monthly Notices of the Royal Astronomical Society, vol. 365, no. 2, pp. 653-672, 2006.

[51] P. E. Boynton, E. J. Groth, D. P. Hutchinson et al., "Optical timing of the crab pulsar, NP 0532," Astrophysical Journal, vol. 175, p. $217,1972$.

[52] J. M. Cordes, "Pulsar timing. II-analysis of random walk timing noise-application to the Crab pulsar," Astrophysical Journal, vol. 237, no. 1, pp. 216-226, 1980.

[53] S. M. Kopeikin, "Millisecond and binary pulsars as nature's frequency standards-I. A generalized statistical model of lowfrequency timing noise ", Monthly Notices of the Royal Astronomical Society, vol. 288, no. 1, pp. 129-137, 1997.

[54] E. J. Groth, "Timing of the crab pular II. Method of analysis," The Astrophysical Journal Letters, vol. 29, p. 443, 1975.

[55] Z. Arzoumanian, "Radio observations of binary pulsars," Bulletin of the American Astronomical Society, vol. 26, p. 888, 1994.

[56] R. M. Canup and W. R. Ward, "A common mass scaling for satellite systems of gaseous planets," Nature, vol. 441, no. 7095, pp. 834-839, 2006.

[57] K. Wada, E. Kokubo, and J. Makino, "High-resolution simulations of a moon-forming impact and postimpact evolution," Astrophysical Journal Letters, vol. 638, no. 2, pp. 1180-1186, 2006.

[58] J. W. Barnes and D. P. O’Brien, "Stability of satellites around close-in extrasolar giant planets," Astrophysical Journal Letters, vol. 575, no. 2, pp. 1087-1093, 2002.

[59] R. C. Domingos, O. C. Winter, and T. Yokoyama, "Stable satellites around extrasolar giant planets," Monthly Notices of the Royal Astronomical Society, vol. 373, no. 3, pp. 1227-1234, 2006.
[60] K. Atobe and S. Ida, "Obliquity evolution of extrasolar terrestrial planets,” Icarus, vol. 188, no. 1, pp. 1-17, 2007.

[61] R. Silvotti, S. Schuh, R. Janulis et al., "A giant planet orbiting the "extreme horizontal branch" star V 391 Pegasi," Nature, vol. 449, no. 7159, pp. 189-191, 2007.

[62] F. Mullally, D. E. Winget, and S. O. Kepler, "Searching for planets around pulsating white dwarf stars," in New Horizons in Astronomy: Frank N. Bash Symposium, vol. 352, p. 265, Austin, Tex, USA, 2006. 

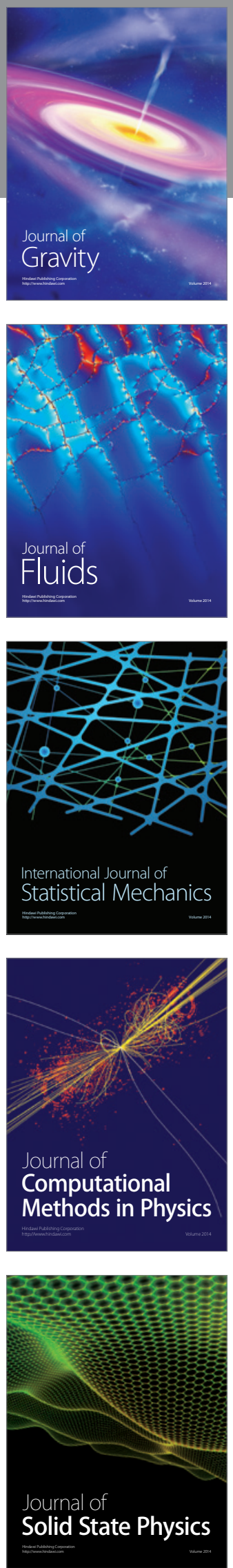

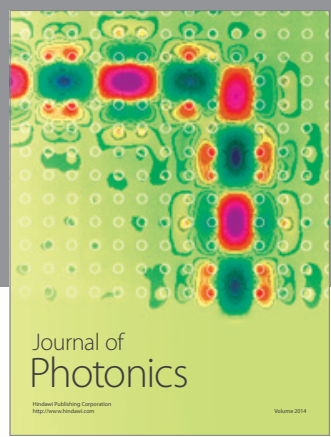

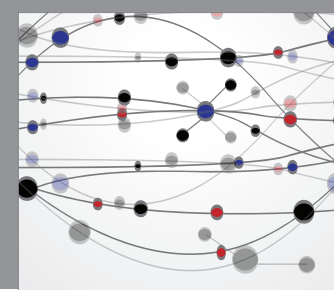

The Scientific World Journal

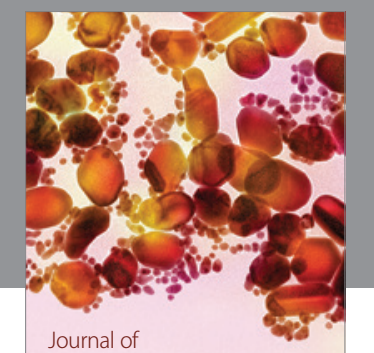

Soft Matter
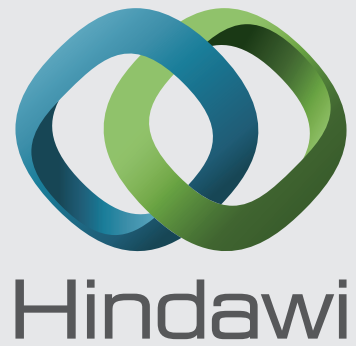

Submit your manuscripts at

http://www.hindawi.com
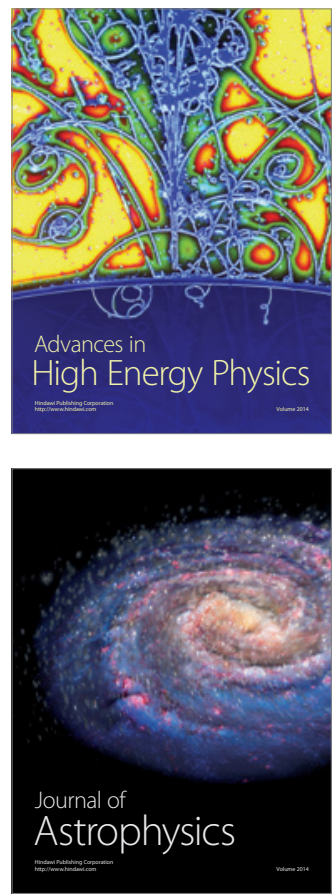
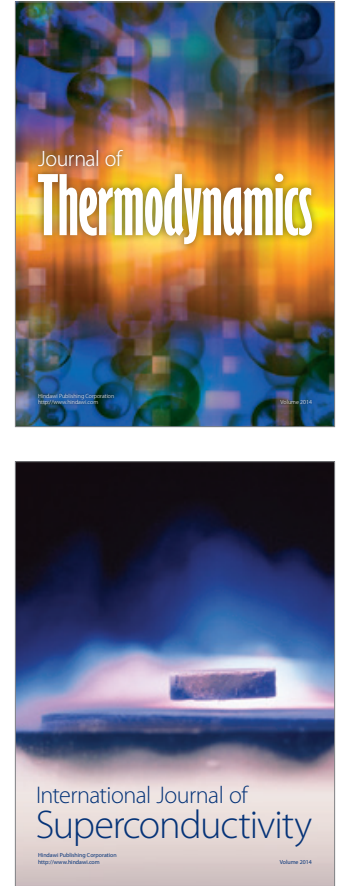
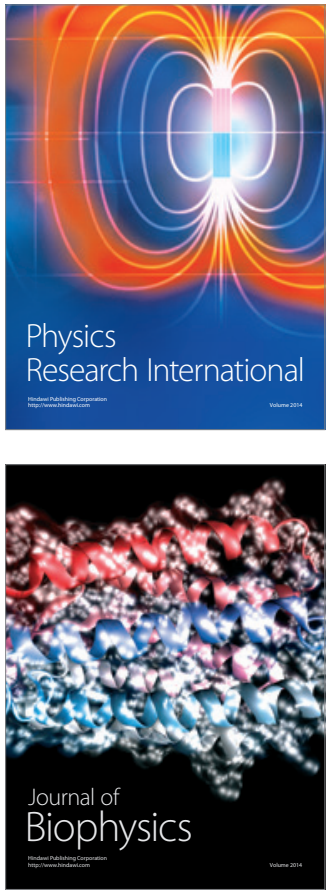
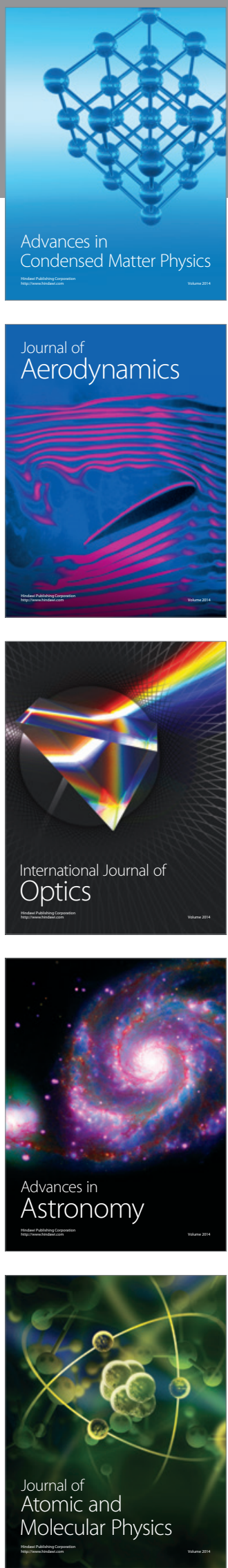